Editorial

\title{
To NET or not to NET
}

\author{
Jadwiga Jablonska \\ Helmholtz Centre for Infection Research, HZI, Inhoffenstr. 7, D-38124 Braunschweig, Germany
}

Do Neutrophils Formneutrophil Extracellular Traps (NETs) as response to single small pathogens or not? Recent findings of Nora Branzk and colleagues (Branzk et al., 2014) questioned previous concepts regarding NETs formation in response to inflammatory stimuli and pathogens.

Neutrophils are one of the first cells of the immune system that are recruited to the site of infection and are equipped with potent antimicrobial properties. Conventional neutrophil-mediated killing involves cell recruitment and phagocytosis of microbes. The process of phagocytosis is triggered when phagocytic receptors recognize the target and stimulate the cell to engulf it. Upon phagocytosis, neutrophil granules fuse with the phagosome. Engulfed microbes are killed by the ensuing oxidative burst and by the discharge of antimicrobial granule contents into the phagocytic vacuole (Urban et al., 2006). This process, however, requires that the target is sufficiently small that it can be engulfed. In case of alarger pathogen neutrophils could bind to it and to degranulate into the extracellular space, in the process called exocytosis. This mechanism was shown already in the early 1970's by Henson and colleagues (Henson 1971). Just in 2004, Brinkmann et al. (2004) discovereda novel third mechanism that could be used to facilitate extracellular killing of pathogens (Brinkmann et al., 2004). This process was called NETosis. During this process neutrophils kill extracellular pathogens while minimizing damage to the host cells. Bacterial pathogens are recognized by the immune system through pathogen-associated molecular patterns that bind Pattern Recognition Receptors (PRRs), with Toll-Like Receptors (TLRs) as the prototypic PRRs being expressed by neutrophils (Mantovani et al., 2011).

Neutrophil extracellular traps consist of nuclear-or mitochondrial-derived web-like DNA strands released from neutrophils. Interestingly, granulocyte homologues in lower vertebrates and even plants are able to release NETs, showing its importance in inflammatory reactions of the host. The NETs comprise histones H1, H2A, H2B, $\mathrm{H} 3$ and $\mathrm{H} 4$, as well as granule proteins, which line the DNA backbone (Brinkmann et al., 2004). They contain proteins from azurophilic granules (neutrophil elastase, cathepsin $G$ and myeloperoxidase) as well as rom specific granules (lactoferrin) and tertiary granules (gelatinase). Once bound to NETs, the associated histones and antimicrobial proteins degrade virulence factors and kill pathogens (Wartha et al., 2007). NETs provide for a high local concentration of antimicrobial components and are used to bindand kill microbes extracellularly, independent of phagocytic uptake.

Complex mechanisms responsible for NET generation have been revealed in vitro. NET formation involves Reactive Oxygen Species (ROS) (Fuchs et al., 2007) and the combined action of elastase and myeloperoxidase (Papayannopoulos et al., 2010). Moreover, it may further involve histone deimination by peptidylarginine deiminase enzymes, autophagy, Rac 2 and signaling through mitogen-activated protein kinases (Hakkim et al., 2011).

Initially, NETs have been consistently shown to bind and kill both Gram-negative and Gram-positive bacteria (Brinkmann et al., 2004). More recently, it has also been reportedthat not only bacteria but also pathogenic fungi such as Candida albicans induce neutrophils to form NETs that capture and kill hyphae as well as yeast-form cells (Urban et al., 2006). Subsequent studies extended this view by showing that NET formation or impaired NET degradation may also have a pathological role in noninfectious conditions, for instance in development of autoimmune diseases like Systemic Lupus Erythematosus (SLE) (Wartha et al., 2007) or inflammatory diseases like autoimmune vasculitis (Kessenbrock et al., 2009), thrombosis (Fuchs et al., 2010) and psoriasis (Lin et al., 2011). Moreover, NETs were also shown to play an important role during metastatic processes, by trapping migrating tumor cells in the microvasculature and promoting micrometastasis formation (Cools-Lartigue et al., 2013).

However, using both phagocytosis and NETosis might be counterproductive if neutrophils engulf bacteria and then release all of their granule contents into the extracellular matrix. The association of NETosis with other antimicrobial functions of neutrophils, such as phagocytosis, has not been clearly defined. Therefore, the mechanism proposed by Branzk et al. (2014) in the current issue of Nature Immunology (Branzk et al., 2014) adds a new aspect to the regulation of the neutrophil biology, but at the same time raises questions. The authors show that neutrophils are able to sense microbe size and accordingly adapt killing mechanisms. Small pathogens, like single small yeasts, are not able to initiate NETs release due to Neutrophil Elastase (NE) sequestration in phagocytic granules. These pathogens are rather phagocytosed. When phagocytosis is successful, granules fuse with phagosomes and elastase is delivered into the phagosome. Since NE migration into nucleus is inhibited, NETosis cannot take place. On the other hand, 
large pathogens, such as Candida albicans hyphae and extracellular aggregates of Mycobacterium bovis,were able to stimulate neutrophils to release NETs. NETosis requires ROS-and myeloper oxidase-facilitated release of elastase from granules into the nucleus, where it cleaves histones to decondense chromatin and initiate the NETosis program. In support of this model, the investigators shown that inhibiting phagocytosis or granule-phagosome fusion leads to the release of NETs in response to a yeast-locked strain of $C$. albicans. This suggests that blocking the trafficking of elastase to phagosomes is sufficient to induce NETosis, independently of the pathogen size.

Whether this size-sensing mechanism also applies to other large pathogens such as multi cellular parasites remains to be determined, but this discovery obviously differs from the findings of Urban et al. (2006) in 2012. In this study the authors demonstrated thatboth yeastform and hyphal cells of C. albicans, are captured and killed by NETs. No blockade of NETs formation was observed in case of small yeast forms of fungi. Moreover, Gram positive and negative bacteria were also shown to be caught by NETs (Urban et al., 2006), regardless of their small size. What could be the reason for this observed dissimilarity is to be resolved.

In addition, the results of Branzkvary from another recent publication in Nature Yipp et al. (2012), which described that live neutrophils are able to simultaneously form NETs while phagocytosing pathogen. Obviously, NET formation was not inhibited by successful phagocytes is and post-phagocytic neutrophils were still able to release NETs. Moreover, NETs were again formed in this case independently of the pathogen size, as responses were observed to living, or even dead, washed bacteria. The authors observed a nuclear neutrophils that contained bacteria, implying that phagolysosome maturation and NET release could be separately compartmentalized (Yipp et al., 2012).

So, it is still an open question whether neutrophils form NETs in response to single small pathogens or not. Another question is whether phagocytosis and NETosis could occur at the same time, in the same cell. What are the exact mechanisms regulating these processes? The evidence on NET generation so far indicates that there could be more than one NET formation machinery relevant for host defense. Obviously, neutrophil extracellular trap development and its effects on neutrophil behavior in vivo are much more complex and dynamic than previously thought. Apparently, neutrophils are capable of using their killing tools in multiple mannersin order to accomplish the most effective protection of the host.

\section{Reference}

Branzk, N., A. Lubojemska, S.E. Hardison, Q. Wang and V. Papayannopoulos et al., 2014. Neutrophils sense microbe size and selectively release neutrophil extracellular traps in response to large pathogens. Nat. Immunol.,15: 1017-1025. DOI: 10.1038/ni.2987
Brinkmann, V., U. Reichard, C. Goosmann, B. Fauler and A. Zychlinsky et al., 2004. Neutrophil extracellular traps kill bacteria. Science, 303: 1532-1535. DOI: $10.1126 /$ science. 1092385

Cools-Lartigue, J., J. Spicer, B. McDonald, S. Gowing and L. Ferri et al., 2013. Neutrophil extracellular traps sequester circulating tumor cells and promote metastasis. J. Clin. Invest., 123: 3446-3458.

DOI: $10.1172 / \mathrm{JCI} 67484$

Fuchs, T.A., A. Brill, D. Duerschmied, D. Schatzberg and D.D. Wagner et al., 2010. Extracellular DNA traps promote thrombosis. Proceedings of the National Academy of Sciences of the United States of America, (USA' 10), New York, pp: 1588015885. DOI: $10.1073 /$ pnas. 1005743107

Fuchs, T.A., U. Abed, C. Goosmann, R. Hurwitz and A. Zychlinsky et al., 2007. Novel cell death program leads to neutrophil extracellular traps. J. Cell Biol., 176: 231-241. DOI: 10.1083/jcb.200606027

Hakkim, A., T.A. Fuchs, N.E. Martinez, S. Hess and H. Waldmann et al., 2011. Activation of the Raf-MEKERK pathway is required for neutrophil extracellular trap formation. Nat. Chem. Biol., 7: 75-77. DOI: 10.1038/nchembio.496

Henson, P.M., 1971. Interaction of cells with immune complexes: Adherence, release of constituents and tissue injury. J. Exp. Med.134: 114-135.

Kessenbrock, K., M. Krumbholz, U. Schonermarck, W. Back and D.E. Jenne et al., 2009. Netting neutrophils in autoimmune small-vessel vasculitis. Nat. Med., 15: 623-625. DOI: 10.1038/nm.1959

Lin, A.M., C.J. Rubin, R. Khandpur, J.Y. Wang and A.T. Bruce et al., 2011. Mast cells and neutrophils release IL-17 through extracellular trap formation in psoriasis. J. Immunol., 187: 490-500. DOI: $10.4049 /$ jimmunol.1100123

Mantovani, A., M.A. Cassatella, C. Costantini and S. Jaillon, 2011. Neutrophils in the activation and regulation of innate and adaptive immunity. Nat. Rev. Immunol., 11: 519-531. DOI: 10.1038/nri3024

Papayannopoulos, V., K.D. Metzler, A. Hakkim and A. Zychlinsky, 2010. Neutrophil elastase and myeloperoxidase regulate the formation of neutrophil extracellular traps. J. Cell Biol., 191: 677-691. DOI: $10.1083 /$ jcb.201006052

Urban, C.F., U. Reichard, V. Brinkmann and A. Zychlinsky, 2006. Neutrophil extracellular traps capture and kill Candida albicans yeast and hyphal forms. Cell Microbiol., 8: 668-676. DOI: $10.1111 /$ j.1462-5822.2005.00659.x

Wartha, F., K. Beiter, S. Normark and B. HenriquesNormark, 2007. Neutrophil extracellular traps: Casting the NET over pathogenesis. Curr. Opin. Microbiol., 10: 52-56. DOI: 10.1016/j.mib.2006.12.005

Yipp, B.G., B. Petri, D. Salina, C.N. Jenne and H.C. Meijndert et al., 2012. Infection-induced NETosis is a dynamic process involving neutrophil multitasking in vivo. Nat. Med., 18: 1386-1393. DOI: 10.1038/nm.2847 